\title{
The effect of Foam Rolling and Dynamic Stretch on some physical abilities of female Handball Players Hala Ahmed Zaky ${ }^{1}$, Maha Mohamed Khalil ${ }^{2}$, Mai Hesham Barakat ${ }^{3}$
}

${ }^{1}$ Professor of Handball, Department of Sports Training, faculty of Physical Education for Girls, Helwan University, ${ }^{2}$ Professor of sports physiology, Department of Biological Sciences and Sports Health, faculty of Physical Education for Girls, Helwan University, ${ }^{3}$ Handball coach with international classification $B$

\section{Abstract:}

The monotonous and strenuous shoulder moving pattern during throwing may result in micro trauma and fibrous adhesions between the layers of fascia among athletes. In the last decade, self myofascial release (SMR) has gained good reputation as a way to treat these adhesions. However, there is limited data demonstrating the effects on upper body performance among athletes.

Aims : The purpose was to examine the effects of an acute bout of upper body SMR with Foam Rolling as a new method for SMR or from dynamic stretch (DS), on shoulder range of motion (ROM), throwing velocity and throwing accuracy. Also to examine correlations between shoulder ROM and throwing velocity/accuracy

METHODS: 20 elite female handball players (age $22.83 \pm 1.52$ years, weight $69.16 \pm 4.62 \mathrm{~kg}$ ) were included in the study. The study had an experimental cross-over design in which participants first performed baseline measures. They also performed SMR and DS in two different trials

The trials were supervised and separated by 6 weeks. During SMR, the athletes performed a series of foam rolling exercises during 5 minutes for the muscles surrounding the shoulder joint. The same muscles were stretched for 5 minutes during the DS trial. Directly after foam rolling and DS, shoulder ROM, throwing velocity and throwing accuracy were measured using a gravity reference goniometer, radar gun and a high speed video camera. ANOVA with repeated measures and t-tests were used to analyze differences between and within groups.

RESULTS: There were no statistically significant differences in either shoulder ROM, throwing velocity or throwing accuracy between the three trials. There was a moderate correlation between shoulder extension and mean accuracy $(r=0.62 ; p=0.004)$ after DS.

CONCLUSION: An acute bout of upper body SMR does not affect ROM and does not improve throwing velocity or throwing accuracy compared to baseline or DS among elite female handball players. Nor could any correlation between ROM and throwing velocity or throwing accuracy be observed.

\section{Introduction}

As a handball player, it is crucial to throw the ball as fast as possible with good accuracy in order to score. A well-executed training program is crucial, whereby coaches and athletes all over the world are trying to refine and optimize training programs in order to increase the athletes' performance (Van den Tillaar, 2004). There is a wide spread use of Self Myofascial Release (SMR) among recreational gym members and professional athletes as a way to improve performance, increase range of motion (ROM) and improve recovery (Castiglione, 2008, Clark \& Russell, 2009 \& Boyle, 2009). During the last five years and 
especially the last two years, there has also been a growing interest within the scientific literature regarding the beneficial effects from SMR on the human body (Curran et al. 2008; Healey et al. 2014; Macdonald et al. 2013, 2014; Sullivan et al. 2013 \& Okamoto et al. 2014). Unexpectedly, there is yet no published research investigating the effects from SMR on athletes as far as we know. Neither is there any research investigating the effects of SMR on the upper body. Therefore, this study is investigating the effects on performance from an acute bout of SMR in comparison with an acute bout of dynamic stretch on elite handball players. If it proves to be beneficial it would be practically applicable and valuable to perform SMR both before and during game play. This study investigates the effects on ROM, throwing velocity and throwing accuracy.

\section{Background}

Handball is an intermittent sport which put a lot of physical demands on the players' aerobic and anaerobic capacity (Póvoas et al. 2012). Players are moving rapidly in all direction across the field, jumping and throwing quickly whereby players need to have strength, agility and endurance to withstand the demands that is being put on them during both practice and game. The main key characteristics in elite handball are endurance, jumping ability, sprint performance and throwing velocity (Kruger et al. 2013). Elite handball players have more experience which is an important factor when it comes to designing training programs, performing physical tests and study design. There is also a need for high levels of aerobic capacity rising towards $60 \mathrm{ml} / \mathrm{kg} / \mathrm{min}$ when heart rates rise above 160 beats $/ \mathrm{min}$ which occurs during sprinting and backwards movement from one court half to the other (Ziv and Lidor, 2009). During one season, each handball player performs at least 48.000 throwing motions with throwing speed approaching $130 \mathrm{~km} / \mathrm{h}$ (Pieper, 1998). This repeated throwing puts great demands on the shoulder joint resulting in a functional instability, which in turn increases the risk for injuries (Gurinder, 2011 and Edouard, et al. 2013). There is, as described by Ziv and Lidor (2009), two possible strategies for scoring: 1) throw the ball as fast as possible without aiming in order to surprise the goal keeper, and 2) throw the ball as accurately as possible to keep the ball out of reach of the goal keeper. Today, most studies have focused towards throwing velocity but have not been looking at accuracy (Ziv and Lidor, 2009). There is as far as we know no study who has been investigating a way to increase throwing velocity and accuracy at the same time. Coaches and scientists have earlier made different attempts in constructing specific training programs (TP) in order to increase the players throwing velocity, resulting in a two to seven percent increase (Van den Tillaar, 2004). However, no conclusions could be drawn but only advice could be given. For example: the specific TP needs to be sustained during five weeks with at least three training sessions per week (Van den Tillaar, 
2004). But there is no acute short term solution for increasing throwing velocity despite the plausible benefits from an increased throwing velocity.

To be able to maximize the athletes throwing velocity without increasing the risk of injuries, there is a need for balance between mobility, muscular balance and dynamic stability around the glenohumeral joint (Borsa et al. 2008). Mobility is needed in order to reach maximum rotation so that velocity can be transferred to the ball, but at the same time, there is a need for stability in order keep the humeral head in the glenoid socket and stabilize the joint. This is known as the "throwers paradox" where the relationship between mobility and stability needs to be maintained in order to reduce the risk of injuries (Braun et al. 2009). Kinematic analysis of handball throwing in a standing position shows that internal shoulder rotation angular velocity is an important contributor to throwing velocity (Van den Tillaar and Ettema, 2004; Ettema, 2007 \& Wagner and Müller E, 2008). However, conflicting findings shows that peak torque of internal and external rotation is not related with ball velocity (Zapartidis, 2007).

The normal ROM around the shoulder among handball players is not clearly investigated, there is however a well-researched area among baseball pitchers which are doing a similar shoulder moving pattern during throws. It is common that baseball pitchers have increased humeral head retroversion in relation to increased humeral external rotation together with a reduced internal rotation in the dominant shoulder (Heber et al. 2002; Osbahr et al. 2002 \& Reagan et al. 2002). Pieper (1998) discussed the fact that the increased humeral retroversion might act as a protection mechanism for the anterior capsulabral complex, since the retroversion allows more external rotation of the shoulder without putting excessive strain on the joint complex (Pieper, 1998). It does however seem as the players overall rotational arc (maximal internal rotation to maximal external rotation) is the same between the dominant and non-dominant shoulder. They also have a similar proprioceptive sensation in the dominant shoulder compared to the non dominant despite changes in internal and external ROM (Safran et al. 2001).

The throwing motion during overhead throwing is very quick and the muscles are sequentially activated to a total period of time up to 120 milliseconds in the kinematic chain during an overarm throw. Thereby it is possible to increase the throwing velocity by increasing thevelocity of the initial movement in the kinematic chain (Grezios et al. 2006). The equation of the distal joint throwing velocity is described as following by Grezios et al (2006)

the absolute speed of the first joint and $\sum \mathrm{VGel} / \mathrm{Di} \mathrm{I}$ is the relative speed of the following joint in relation to the proximal joint. Since the muscles are sequentially activated, an increased velocity of the proximal movement would lead to an increased throwing velocity. During the first 50 milliseconds of the throw, the 
stretch shortening-cycle (SSC) is apparent (Grezios et al. 2006). A passive muscle does not exhibit elastic characters and must be pre-activated in order to maximize the effect from the SSC. An increased pre-activation enables the muscles to store more elastic energy and increase the amount of SSC (Grezios et al. 2006) which in turn could lead to an increased throwing velocity. Since internal rotation is important for throwing velocity, it is important to emphasize and maintain a good ROM for shoulder internal rotation among handball players. The cause for the loss of internal rotation among handball players is a multifactorial matter where a number of different factors affect the ROM. Reactive scarring of the contracture of the posterior shoulder capsule with a resulting immobility of the posterior shoulder could be one reason (Borsa et al. 2008). The reactive scarring forms over time when multiple micro traumas with resulting scars in the connective tissue occurs, thereby creating adhesions between layers of connective tissue. Another explanation for the overall changes in ROM is the involvement of the fascia surrounding the muscles and connecting the joints to each other. Stiffness of the fascia as a result of monotonous movement patterns with resulting micro trauma. This micro trauma may lead to an inflammatory response which in turn leads to fascia scar tissue with resulting adhesions between the fascia and muscle. These adhesions are one possible explanation for changes in ROM and muscular dysfunction (Cantu and Grodin, 2001 \& Curran et al. 2008).

Fascia is more or less inseparable from all structures in the body and acts as a connecting structure linking body tissues to each other in order to enhance function and stability in the body. A more recent approach to the area of mobility and its effect on athletic performance is the fascia as a major player where the fascia acts as a tissue memory, adapting to all the movements in the body (Paoletti, 2006). This "cellular memory" is being described by Paoletti (2006) as something that is being derived from the embryonic growth and that this "cellular memory" acts as a tissue memory that registers any deformation which they undergo andcorrects the deformations. The ability to correct deformations is however sooner or later exceeded if the structure deformation is too extreme, whereby a progressive pathology with continual changes in the structure occurs. These continual changes are shown to induce a fibrous adhesion between the different layers of fascia, preventing adjacent dense layer of tissue to glide past one another which in turn prevents normal muscle mechanics (i.e. decrease strength, endurance and ROM) (Curran et al. 2008 \& Graham et al. 2013). Other authors have found the same properties for the fascia and states that the fascia is able to adapt fiber arrangement, density and length according to local demands (Findley, 2009). Within the fascia, there are a large number of mechanoreceptors, which means that the fascia plays a large role in nociception and proprioception (Yahia, 1992 \& Van der Wal, 2009). 
Majority of the input to these mechanoreceptors arrive from the interstitial receptors that are directly connected to the autonomic nervous system (ANS). Thereby, stimulation of the intra fascial mechanoreceptors changes the input to the ANS resulting in a changed tonus regulation of the motor units connected to the affected tissue. The result is a more relaxed and pliable tissue, leading to a larger mobility (Schleip, 2003).

\section{Dynamic Stretch}

Dynamic stretching (DS) is a mobility drill that involves a controlled movement trough the active ROM for a joint (Fletcher, 2010). Research has showed that an acute bout of low-volume DS results in a significant decrease in maximal isometric peak force for pectoralis major and triceps brachii. However, no significant changes in average amplitude of surface EMG, time to maximal isometric force or rate of force production were found (Leone et al. 2012). Overall, literature indicates that shorter durations of DS does not inhibit muscular performance compared to static stretching and is often recommended during warmup since the movements used are similar to movements that occur during sports (Torres et al. $2008 \&$ Behm and Chaouachi, 2011)

\section{Self Myofascial Release (Foam rolling)}

Self Myofascial Release (SMR) is a modern technique that has been developed from Myofascial Release therapy (MFR) which was a manual-therapy technique developed by Barnes (1997) to reduce fibrous adhesion between the layers of fascia (Barnes, 1997). The main difference between SMR and MFR is that instead of a therapist that provides manual therapy, subjects practicing SMR use their own body mass on a foam roller and/or a dense ball in order to isolate specific areas and treat soft tissue restrictions (Curran et al. 2008 \& Castiglione, 2010). Thereby 6 the term "Self Myofascial Release" is applied to techniques that use tools such as foam rollers, tennis balls and other rollers with varying shapes and hardness. The tool is used in order to elongate muscle fibers and fascia while at the same time comprise the area and create an ischemic compression just like acupressure or massage. Thereby increase soft tissue temperature and restore muscle length-tension relationship prior to a workout (Clark and Russell, 2009). The exact mechanism of how this work is not clearly investigated. One hypothesis is that the ischemic compression would loosen up the possible adhesions between layers of fascia and thereby restore soft tissue mobility. Another possible hypothesis was first described by Klinger et al. (2004) which showed in vitro that the stiffness of the fascia is partly due to its water content and when the fascia is comprised, the water is being extruded from the tissue and thereby making the tissue more pliable (Klinger et al. 2004). A more recent study confirmed this results and showed that water returned to the tissue 
within a few hours in a higher concentration than before the water efflux, resulting in a stiffer tissue as a result of the "super compensation" of fluid (Schleip et al. 2012). The period between when the water is being extruded and prior to its refilling are described by Schleip et al. (2012) as a "window of opportunity" for increased mobility/flexibility.

SMR seems to become more frequently used as a warm up-, rehabilitationand/or mobility technique to help promote soft-tissue extensibility, increase ROM and promote muscle function. Layman's literature supports that SMR is commonly used in training facilities by ordinary training facility members but also by coaches and athletes. It is used both before and after training in order to reduce any possible dysfunctions resulting from micro trauma and thereby be able to increase the athletes training volume (Castiglione, 2008, Boyle, 2009 \& Clark and Russell, 2009). There have as far as I know, been six peer reviewed articles investigating the effects of foam rolling before and after exercise / performance test (Curran et al. 2008; Healey et al. 2014; Macdonald et al. 2013, 2014; Okamoto et al. 2014 \& Sullivan et al. 2013). Curran et al. (2008) showed that myofascial rollers made of harder material was more beneficial compared to softer, in order to optimize muscle function (Curran et al. 2008). Two articles found that an acute bout of SMR increased lower body ROM without a subsequent decrease in muscle activation or force (MacDonald et al. 2013 \& Sullivan et al. 2013). One article found that foam rolling prior to exercise was beneficial in improving dynamic movement, muscle activation, ROM and reduced muscle soreness in comparison to a control group (MacDonald et al. 2014). Another article found no difference between foam rolling and planking on athletic performance. However, the post-exercise fatigue was significantly reduced after foam rolling 7 compared to planking. This reduced feeling of fatigue could in turn augment continuous performance enhancements (Healey et al. 2014). Okamoto et al. (2014) investigated the effect of SMR on vascular endothelial function and arterial stiffness and found that one bout of SMR reduced arterial stiffness and improved vascular endothelial function compared to a control group (Okamoto et al. 2014). These findings support the layman's literature and the peerreviewed studies report consistent findings that SMR may increase one or more physiological parameters that may augment athletic performance. However, there is a lacking consistency in methods and materials that has been used in the existing articles investigating different aspects of SMR. Even though the earlier findings support the fact that SMR increases performance parameters in one way or another, there is a various amounts of conclusions and explanations for why this happens. The inconsistency regarding methods and materials used is thought to be one reason for the diverse conclusions among studies.

There is a growing interest for SMR and its possible benefits on human 
performance and mobility. However, none of the published studies have been performed on athletes and most of the published studies are aiming towards effects on the lower extremities. Empirical evidence and literature investigating the effect of SRM for the upper body is lacking. Therefore, the purpose of this study was to examine and evaluate if an acute bout of SMR or DS could have an effect on ROM, throwing velocity and/or accuracy among elite female handball players.

\section{Aims of study}

The study aims to investigate the following:

- The effect of foam rolling on some physical abilities to female handball players as (throwing velocity, accuracy and range of motion of shoulder joint ).

- Comparison between the effect of foam rolling and dynamic stretching exercises .

\section{Study hypotheses}

1) There are significant differences between the pre and post measurement of the SMR trial experimental group to throwing velocity and/or accuracy.

2) There are significant differences between the pre and post measurement of the DS trial experimental group to throwing velocity and/or accuracy.

3) There are significant differences between SMR and DS on throwing velocity and/or accuracy.

4) There are significant differences between SMR and DS to increase shoulder ROM in the shoulder joint?

5) There is a correlation between shoulder ROM and throwing velocity and/or accuracy.

\section{Methods}

Subjects

The female players were at the moment playing in the Egyptian handball elite series and Champions League. A total of 20 healthy female participants (age $22.83 \pm 1.52$ years, weight $69.16 \pm 4.62 \mathrm{~kg}$ ) met inclusion and exclusion criteria.

\section{TABLE 1}

Descriptive statistics of participants

$(n=20)$

\begin{tabular}{cc}
\hline Variables & Mean \pm SD2 \\
\hline Age (years) & $22.83 \pm 1.52$ \\
\hline Weight $(\mathrm{kg})$ & $69.16 \pm 4.62$ \\
\hline Length $(\mathrm{m})$ & $173.16 \pm 8.02$ \\
\hline BMI $(\mathrm{kg} / \mathrm{m})$ & $23.3 \pm 1.94$ \\
\hline Experience (years) & $11.75 \pm 1.05$ \\
\hline Training frequenency (hour/week) & $11 \pm 0.54$
\end{tabular}

'BMI - Body Mass Index; SD - Standard Deviation 


\section{Testing Procedures}

In an experimental, cross-over design, the experiment was carried out during the athletes' regular scheduled training and consisted of three measurement occasions. One baseline measurement trial and two experimental trials (DS and SMR) were performed. All subjects were split after the baseline trial into either start with the DS-trial or the SMR-trial without any specific order. All trials occurred in the afternoon at the same time separated by 6 weeks with at least $24 \mathrm{~h}$ of rest from any type of heavy exercise prior to each testing occasion in order to minimize shoulder fatigue as a confounding factor (Carpenter et al. 1998). During all trials, measurement of active ROM throughout sagittal shoulder flexion and extension, and internal- and external shoulder rotation in $90^{\circ}$ abduction were measured with a gravity-reference goniometer, (RR Parir, Bålsta, Sweden (Myrin)). Prior to each measurement, all subjects performed a general warm-up consisting of easy jogging and football playing for approximately ten minutes. Thereafter they were asked to lie down in a supine position on a bench with their dominant arm hanging outside the bench, making it able to move freely during measurements. Two examiners performed the measurements where one examiner was in charge of stabilization of the shoulder by applying a posterior-directed force against the subject's clavicle with the heel of the hand, preventing scapula from moving. This method has previously been shown to increase the reliability during ROM-measurement of internal- and external rotation of the shoulder (Awan et al. 2002). The other examiner measured active ROM during sagittal shoulder flexion, extension and thereafter, internal- and external shoulder rotation. Subjects were instructed to perform a full active shoulder flexion starting in a neutral position.

Measurements were taken when subjects had reached their maximal active ROM. Thereafter, they performed a full active shoulder extension starting in a neutral position. Here as well, the measurement was taken when the subjects reached their maximal active ROM. They were told to keep their elbow fully extended during both shoulder extension and flexion. Subjects were then instructed to have their shoulder in $90^{\circ}$ abduction and the elbow in $90^{\circ}$ flexion when they performed a maximal active internal rotation followed by active external rotation. All measurements were performed in a supine position and the readings were taken during the first attempt in order to avoid influences on ROM from repeated testing. The Myrin goniometer was attached with Velcro straps on a rubber cloth to the subjects forearm directly below processus styloideus radii. Myrin goniometer is an inclinometer that has been previously used in a randomized controlled study (Ryans et al. 2005) and has also been shown to be reliable and show good agreement to a three-dimensional ultrasound motion device (Zebris) during measurement of cervical ROM (Malmström et al. 2003). 
Directly after the ROM measurements, subjects performed three seven meter throws with a standard International Handball Federation (IHF) Size 2 handball for women from penalty throw line towards a standard team handball goal $(3 \times 2$ meter). Subjects were told to be in contact with the ground with their front foot during throwing; they were also told to use handball glue during all throws as during game play. Maximal ball velocity (V0) was measured using a radar gun (Bushnell Velocity Radar Gun, Bushnell Outdoor Products TM ®, Overland Park, Kansas, USA) (Caliskan and Akkoyunlu, 2011 \& Cerrah et al. 2011). The radar gun was placed behind the goal aiming at the center of a circular target with 66.5 centimeter in diameter placed in the goal about 1.5 meter above ground (Figure 2). This is a previously used set up that has been shown to be both valid and reliable (Finnoff et al. 2002 \& Skoufas et al. 2003). Subjects were instructed to hit the center of the circle without emphasizing accuracy in order to avoid influencing their throwing speed (Van Den Tillaar and Ettema, 2003; Garcia et al. 2013). Throwing accuracy was measured with a high speed video camera recording at 240 frames per second (fps), (Canon IXUS 220 HS, Canon Svenska AB, Solna, Sweden) placed one meter behind the center of the circle. The placement where the ball passed through the circle was measured at the moment when the ball struck the net. The data was analyzed with Dartfish video analysis software (Dartfish®) to identify the deviations of throws with respect to the center of the circle.

Throwing speed and accuracy were measured during three subsequent throws, after which mean and peak throwing velocity were calculated. If the ball struck the net further away than 5511 centimeter from the center of the circular target, subjects were allowed another throw until they had three approved throws. All three throws were used to calculate mean accuracy, and the best hit was used to estimate peak accuracy.

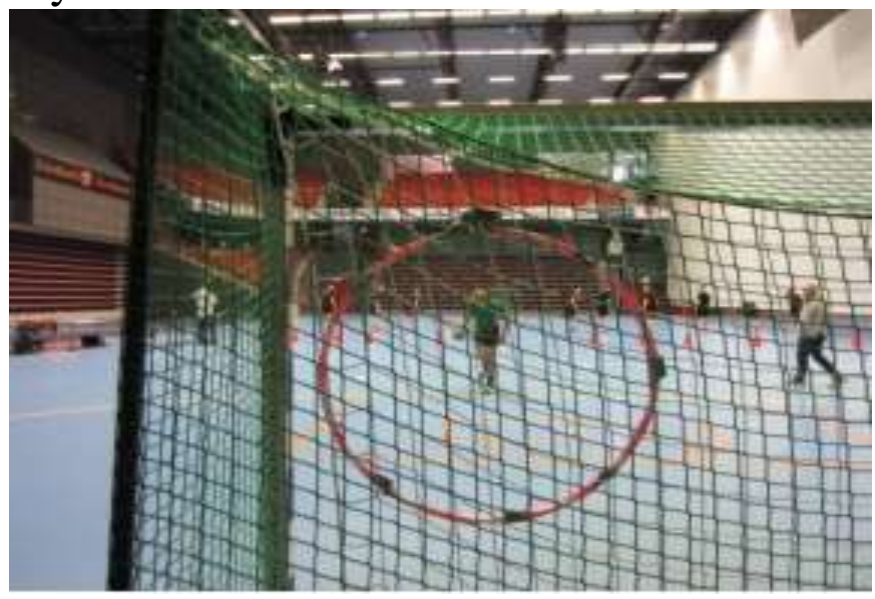

Figure (1)

Throwing accuracy and velocity setup 


\section{Self Myofascial Release}

All subjects performed both experimental trials separated by 6 weeks in a crossover design. The type of SMR that was used was foam rolling. During the SMR-trial, subjects were first instructed in how to use the equipment for the posterior shoulder capsule, m.latissimus dorsi, m.teres major/minor, m.pectoralis major/minor, m.infraspinatus, m.m.rhomboideus major/minor and the thoracic spine. Thereafter they performed 5 different foam rolling exercises for 60 seconds / exercise ( 5 minutes total). The equipment used was a 330 millimeter long foam roller made of a hollowed polyvinyl chloride (PVC) pipe (four millimeter wall thickness) with 110 millimeter in diameter surrounded by a 15 millimeter thick layer of Ethylene-vinyl acetate (EVA) foam (THE GRID, Trigger point Performance Therapy, Texas, USA). During the first exercise targeting the muscles crossing the armpit (m.latissimus dorsi and m.teres major/minor), subjects were instructed to place their body on the side on the foam roller. Their throwing arm was outstretched overhead with the hand behind the neck and their other hand put on the hip (Figure 3). They were then starting at the proximal aspect of humerus and started to roll down the lateral side of the thorax in a speed of about two centimeters / second before turning back and returning towards the proximal part of humerus.

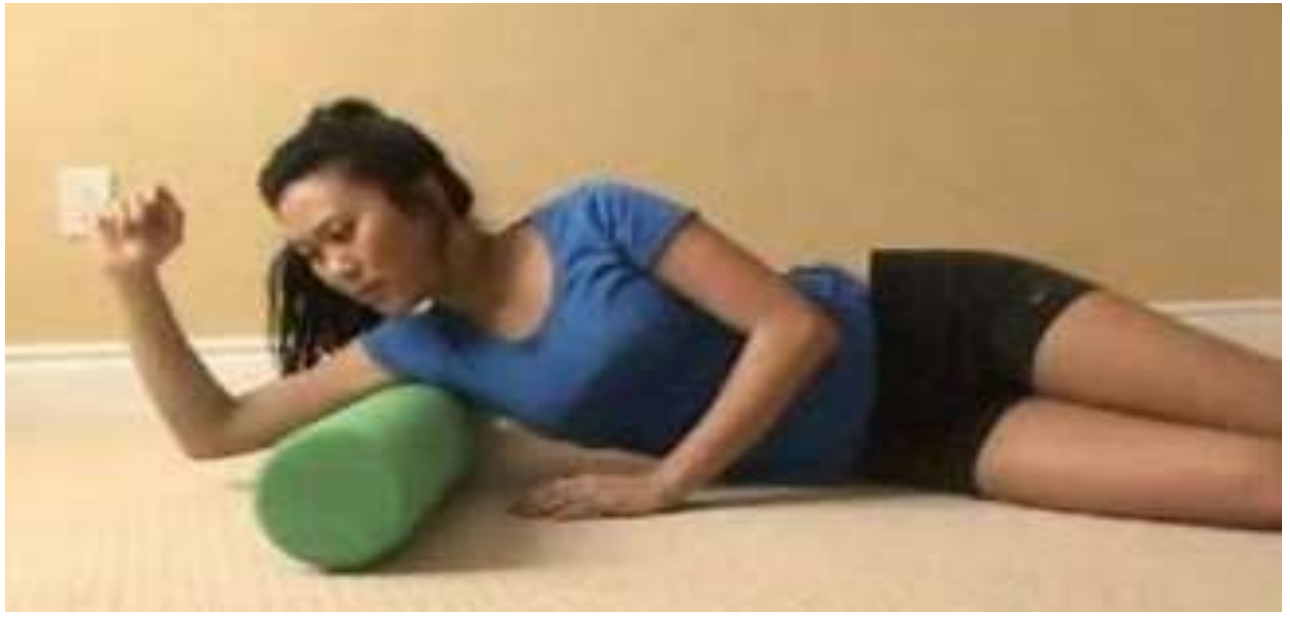

During exercises, Subjects were told to put as much of their bodyweight as possible on the foam roller during all exercises, however pressure was selfadjusted by using hands and feet to offset weight as required. Subjects were instructed to keep a steady pace and quickly turn back to the initial position in one fluid motion. The treatment was supervised by an examiner to ensure proper execution. ROM, throwing accuracy and throwing velocity was measured as during baseline directly after the SMR.

\section{Dynamic Stretching}

The DS group performed the supervised DS for 60 seconds / exercise (5 
minutes total) for the same muscles as treated during SMR. They performed Sleeper stretch, Phelps stretch, overhead backward arm swings and window washers in that order (Appendix 2). These DS exercises were used to stretch the following muscles of the upper body: m.latissimus dorsi, m.pectoralis major/minor, m.m.rhomboideus major/minor, m.deltoideus and m.teres major/minor. After the DS session, the same ROM-measurements and throwing procedure as during the baseline test were performed. All subjects were told not to use SMR at home during the study period. They were also told to avoid any extra stretching except for that normally performed during training.

\section{Ethical and Social Considerations}

The result is presented on group level, meaning that none of the results is possible to derive to a certain person. The findings from this study will help us by giving more information about the possible effects from SMR in comparison with DS both frequently used in training programs for athletes. It will also aid to the health for the population, where an extended knowledge regarding warm up would help both trainers and physiotherapists to affect and formulate more efficient training programs for the general population

\section{Statistical Analyses}

A power analysis was performed before study start to estimate the sample size. The result from the analysis showed that with the alpha level set at $p \leq 0.05$ for statistical significance, and a premise that a five to ten degree change in ROM would be similar to the one presented in other comparable studies, there needed to be at least ten subjects included in this study for a power value of $80 \%$. Data was collected using a Microsoft Excel 2010 spread sheet and is expressed as mean \pm standard deviation (SD). Statistical analyses were performed using Statistica software (IBM SPSS ver.20, Chicago, IL, USA). All data were normally distributed determined 14 by Shapiro-Wilks which could not show a significant difference between groups. A two-way analysis of variance (ANOVA) with repeated measures was performed on all dependent variables. A student t-test was used to analyze changes within the group. The alpha level was set at $p \leq 0.05$ for statistical significance. Pearson product correlation was used to determine any relationship between dependent variables (Vincent and Weir, 2012). The correlation was determined as moderate when $r=0.5-0.8$ and large when $r>0.8$ (Cohen, 1988)

\section{Results}

Throwing Velocity There was no significant difference in peak throwing velocity $(\mathrm{p}=0.74)$ or mean throwing velocity $(\mathrm{p}=0.75)$ between the three trials (Table 2, Figure 4). 


\section{TABLE (2)}

Comparison of throwing velocity among participants between trials Values presented as mean \pm SD

\begin{tabular}{ccccc} 
& & & $(\mathbf{n}=20)$ \\
\hline Variable & Baseline $(\mathbf{k m} / \mathbf{h})$ & DS' $^{\prime}(\mathbf{k m} / \mathbf{h})$ & $\mathbf{S M R}^{\mathbf{2}}(\mathbf{k m} / \mathbf{h})$ & p-value \\
\hline Peak Velocity & $84.80 \pm 8.32$ & $84.55 \pm 7.54$ & $85.15 \pm 7.76$ & 0.74 \\
\hline Mean Velocity & $82.17 \pm 8.13$ & $82.00 \pm 7.68$ & $82.52 \pm 8.02$ & 0.75
\end{tabular}

'DS - Dynamic Stretch, ${ }^{2}$ SMR -Self Myofascial Release

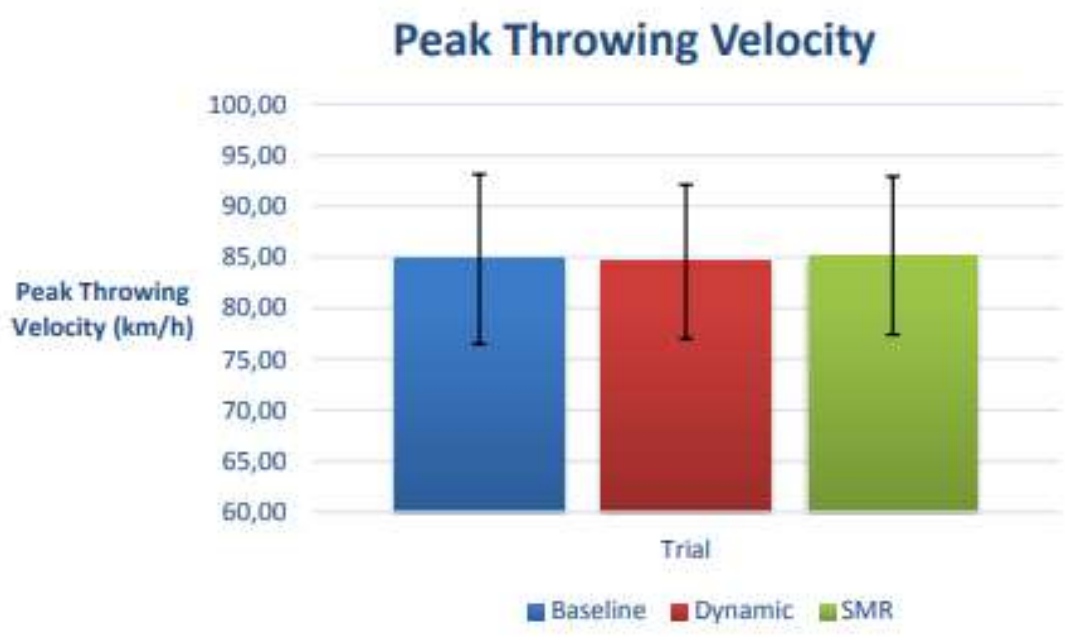

Figure (4)

Peak throwing velocity for all participants $(n=20)$ during baseline, dynamic stretch and SMR Values are presented as means for all participants.

Error bars shows the standard deviation

\section{Throwing Accuracy}

There was no significant difference in peak throwing accuracy $(\mathrm{p}=0.81)$ or mean throwing accuracy $(\mathrm{p}=0.69)$ between the three trials (Table 3, Figure 5). An increased value shows that the handball struck the net further away from the center of the target, thereby indicating a decreased accuracy.

\section{Table (3)}

Comparison of throwing accuracy for all participants between the three trials.

Values are presented as mean \pm SD and show the distance from the center of the target

\begin{tabular}{ccccc} 
& & & $(\mathrm{n}=20)$ \\
\hline Variable & Baseline $(\mathbf{c m})$ & DS' $^{\prime}(\mathbf{c m})$ & SMR $^{2}(\mathbf{c m})$ & p-value \\
\hline Peak Accuracy & $20.70 \pm 12.19$ & $21.20 \pm 13.71$ & $19.10 \pm 9.29$ & 0.81 \\
\hline Mean Accuracy & $32.72 \pm 11.16$ & $35.30 \pm 10.64$ & $33.35 \pm 8.19$ & 0.69 \\
\hline
\end{tabular}

'DS - Dynamic Stretch, ${ }^{2}$ SMR -Self Myofascial Release 


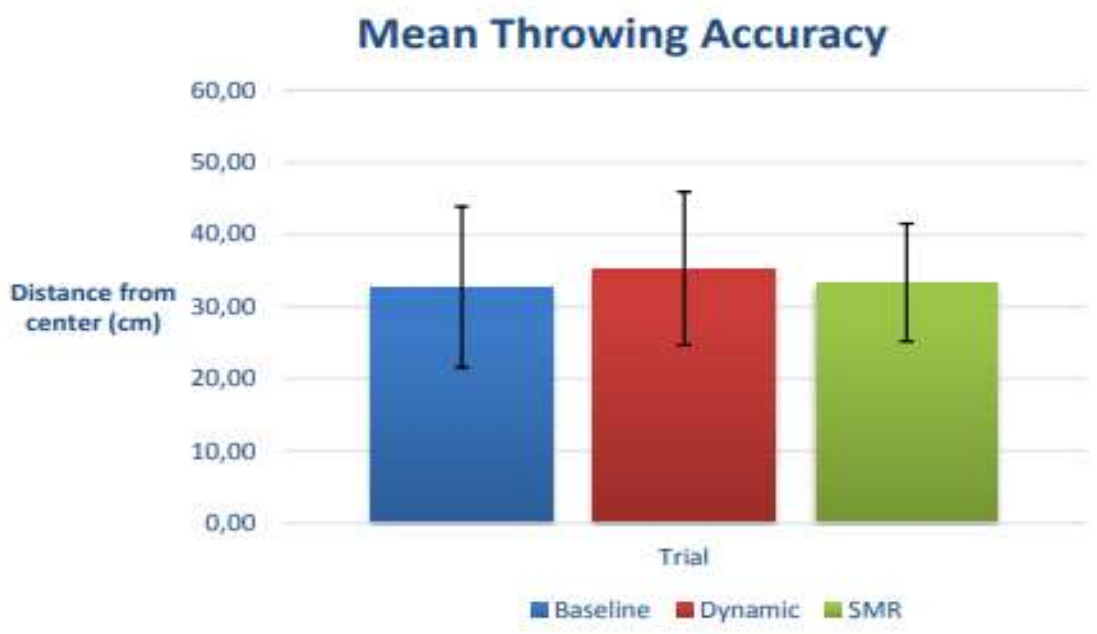

Figure (5)

Mean throwing accuracy (distance from center of target) during baseline, dynamic stretch and SMR Values are presented as means for all Shoulder Joint ROM

participants. Error bars shows the standard deviation

There was no significant difference between the three trials for either flexion $(\mathrm{p}=0.11)$, extension $(\mathrm{p}=0.13)$, internal rotation $(\mathrm{p}=0.51)$ or external rotation $(\mathrm{p}$ $=0.13)$ (Table 4) between all participants.

Table (4)

Comparison of active Range of Motion (degrees) for all participants

between trials. Values presented as mean $\pm \mathrm{SD}$

'DS = Dynamic Stretch, SMR $={ }^{2}$ Self Myofascial Release, ${ }^{3}$ In-Rot $=$ Internal Rotation: ${ }^{4}$ Ex-Rot $=$ External Rotation

\section{Correlation between ROM and Throwing Velocity/Accuracy}

Shoulder extension and mean throwing accuracy measured after DS showed a moderate correlation $(\mathrm{r}=0.618 ; \mathrm{p}=<0.00)$ indicating that players with greater shoulder extension had a lower mean accuracy than players with a more limited shoulder extension after DS. Otherwise there were no moderate or high correlations between any ROM-values and throwing accuracy or throwing velocity during baseline, DS or SMR (Table 5, Table 6). 
Table (5)

Correlation (r) between Range of Motion (degrees) and Throwing Accuracy / Velocity after Dynamic Stretch (DS) for all participants

$(\mathrm{n}=20)$

\begin{tabular}{ccccccccc}
\hline Variable & $\begin{array}{c}\text { Peak } \\
\text { Velocity } \mathbf{r}\end{array}$ & $\mathbf{P}$ & $\begin{array}{c}\text { Mean } \\
\text { Velocity } \mathbf{r}\end{array}$ & $\mathbf{P}$ & $\begin{array}{c}\text { Peak } \\
\text { Accuracy } \mathbf{r}\end{array}$ & $\mathbf{P}$ & $\begin{array}{c}\text { Mean } \\
\text { Accuracy } \mathbf{~}\end{array}$ & $\mathbf{P}$ \\
\hline Flexion & 0.078 & 0.74 & 0.081 & 0.74 & 0.155 & 0.514 & 0.223 & 0.34 \\
\hline Extension & -0.260 & 0.27 & -0.249 & 0.29 & 0.381 & 0.098 & $0.618^{*}$ & 0.00 \\
\hline In-Rot & -0.245 & 0.30 & -0.216 & 0.36 & -0.314 & 0.177 & -0.152 & 0.52 \\
\hline Ex-Rot & 0.179 & 0.45 & 0.140 & 0.56 & 0.194 & 0.413 & 0.087 & 0.72 \\
\hline
\end{tabular}

In-Rot = Internal Rotation: Ex-Rot $=$ External Rotation, $*=$ Moderate Correlation

Table (6)

Correlation (r) between Range of Motion (degrees) and Throwing Accuracy / Velocity after Self Myofascial Release (SMR) for all participants

\begin{tabular}{ccccccccc}
\hline Variable & $\begin{array}{c}\text { Peak } \\
\text { Velocity } \mathbf{~}\end{array}$ & $\mathbf{P}$ & $\begin{array}{c}\text { Mean } \\
\text { Velocity } \mathbf{~}\end{array}$ & $\mathbf{P}$ & $\begin{array}{c}\text { Peak } \\
\text { Accuracy } \mathbf{~}\end{array}$ & $\mathbf{P}$ & $\begin{array}{c}\text { Mean } \\
\text { Accuracy } \mathbf{~}\end{array}$ & $\mathbf{P}$ \\
\hline Flexion & -0.029 & 0.90 & -0.040 & 0.87 & 0.297 & 0.20 & 0.459 & 0.04 \\
\hline Extension & -0.155 & 0.51 & -0.208 & 0.38 & 0.276 & 0.24 & 0.297 & 0.20 \\
\hline In-Rot & -0.004 & 0.99 & -0.032 & 0.89 & -0.154 & 0.52 & -0.242 & 0.30 \\
\hline Ex-Rot & -0.222 & 0.35 & -0.292 & 0.21 & -0.034 & 0.89 & 0.196 & 0.41 \\
\hline
\end{tabular}

In-Rot $=$ Internal Rotation: Ex-Rot $=$ External Rotation

\section{Discussion}

To our knowledge, this is the first study to examine the effects of SMR on athletes. It is also, as far as we know the first study to examine the effects of SMR on the upper body and compare the results to a similar session of DS. The results show that 5 minutes of SMR or DS for the involved muscles during overhead throwing have no significant effect on throwing velocity or throwing accuracy compared to baseline values for all participants. The results also shows that the active shoulder ROM after an acute bout of SMR or DS does not differ significantly compared to baseline values among the elite handball players in this study. Even though the shoulder ROM does not differ between trials, there was a moderate correlation between shoulder extension and mean accuracy after DS, indicating that the greater shoulder extension, results in a decreased mean accuracy after DS. These results are not causal but might indicate that trying to increase the players shoulder extension with mobility exercises such as DS could be counterproductive and lead up to a reduced accuracy among elite handball players.

Healey et al. (2014) used 30 seconds of SMR / muscle group for the lower limb and back and found no significant improvements in the performed athletic tests. Neither could MacDonald et al. (2013) find any significant differences in any 
neuromuscular performance measurements (muscle force, rate of force development and muscle activation) post 2, 1-minute trials of SMR for quadriceps. Sullivan et al. (2013) found no difference in MVC between SMR and the control group. However, twitch force decreased $7.1-10.5 \%$ from pre- to post-SMR. MacDonald et al. (2014) measured vertical jump height and maximal voluntary contractile force which did not change while rate of force development and twitch force did both decrease by $23 \%$ and $14 \% 24$ hour post SMR

Throwing velocity or accuracy did not change for the subjects in this study which goes in line with the small or nonexistent differences in muscle contractile properties seen in earlier studies. The studies performed on DS and muscular performance by Torres et al. (2008) and Behm and Chaouachi, (2011) both shows that shorter durations of DS does not inhibit muscular performance. However, Leone et al. (2012) found that DS decrease maximal isometric peak force for pectoralis major and triceps brachii but does not affect the rate of force production which is more interesting during a dynamic movement. These results from earlier studies are in line with the results for throwing velocity and throwing accuracy between baseline and DS in 23 this study. No other studies have compared SMR to DS regarding athletic performance properties. This study is the first to investigate this and the results shows that there is no difference in throwing performance between SMR and DS.

The results in ROM for the subjects in this study is not directly comparable to results achieved in other studies because of differences in training experience between subjects and the time used during SMR. Sullivan et al. (2013) performed a five second SMR-session and a ten second SMR-session for the hamstring which increased hamstring and lower back ROM by $4.3 \%$ and $6.6 \%$ as measured by a sit and reach test. MacDonald et al. (2013) achieved a $12.7 \%$ (10.6 degrees) improvement in passive knee joint ROM compared to baseline two minutes post 2, 1- minute trials of SMR for quadriceps. MacDonald et al. (2014) did not measure ROM directly after SMR. However, they did not achieve a significant difference in passive ROM for the quadriceps and hamstrings 24 hour post five SMR exercises performed for two minutes each. The measured differences are small but it seems as if a longer duration of SMR is more beneficial in order to increase ROM directly after SMR and that the improvements in ROM decreases by time.

There was a moderate correlation between shoulder extension and mean throwing accuracy measured after DS but no other correlations could be found. The difference in ROM between the trials shows that their overall rotational arc (internal rotation to external rotation) remains about the same during all trials (Baseline, $142^{\circ}$; DS, $142^{\circ} \&$ SMR, $143^{\circ}$ ). Earlier studies have investigated the correlation between external rotation and throwing velocity and found that there 
usually is a correlation between an increased external rotation and increased throwing velocity. There was however no difference in any measured ROM for the subjects, thereby, there was neither any correlation between external rotation and throwing velocity as shown by Van den Tillaar and Etterna $(2004,2007)$ \& Wagner and Müller E (2008). There is a possibility that the overall rotational arc is a more important contributor to throwing velocity compared to internal- or external rotation only. Further studies is needed to confirm this hypothesis.

Neither ROM, throwing velocity or accuracy differed significantly between SMR and DS. However, it would be interesting to record muscle activation with surface electromyography (sEMG) to see if the activation of the stabilizing muscles surrounding the shoulder joint increased. Grezios et al. (2006) stated that in order to maximize the effect from SSC, the muscle must be pre-activated. Although the throwing velocity did not increase, the overall stability around the shoulder joint might have increased and thereby leading to a reduced risk of injuries as discussed by Gurinder (2011) \& Edouard et al. (2013). Further studies measuring muscle activation with EMG around the shoulder joint is needed in order to control for this.

\section{Method Discussion}

The overhead throwing motion involves a lot of muscles which is sequentially activated in order to maximize throwing velocity. At the same time, a lot of muscles around the scapula work synergistically to the agonist and antagonist muscles during an overhead throwing motion. This complexity makes it difficult to perform the myofascial release with a foam roller on a single muscle. There is probably more beneficial to use a smaller tool such as a tennis ball to reach and affect each muscle individually.

One possible explanation for the effects in muscle-length tension that has been observed earlier could be the increased soft tissue temperature (Clark and Russell, 2009). In order to exclude the increased soft tissue temperature as a confounding factor in this study, the DS group was used as a control group since the DS would increase soft tissue temperature in a similar way as SMR (Behm and Chaouachi, 2011). However, SMR and DS do affect the soft tissue in different ways and the two types of soft tissue mobility exercises are not directly comparable.

The abnormal crosslink and scar tissue formation within the fascia surrounding the shoulder joint are most likely to be found among elite handball players as a result from repeated strenuous movement. SMR works mechanically by applying a direct pressure towards soft tissue. The hypothesis is that this applied direct pressure would be enough to shear out the scar tissue and abnormal crosslinks and reduce fibrous adhesion between the layers of fascia. Whether the 
26 fascia becomes more pliable as a result of changed tonus regulation, proposed by Schleip (2003) and/or due to the extruded water after the compression showed by Klinger et al. (2004) and Schleip et al. (2012) is yet to be determined. However, the duration of mechanical stress application from this treatment may have been too short in order to elicit any significant changes despite a sufficient pressure according to earlier studies where it has been calculated that mechanical stress forces ranging from $24-115 \mathrm{~kg}$ would be high enough to cause a more pliable tissue (Threlkeld, 1992).

The theory described by Schleip et al. (2012) propose that the fascia acts as a sponge with a "window of opportunity" for increased mobility/flexibility during the time where the water is being extruded during SMR and before its refilling is interesting. The result from this study did not show any increases in ROM. That is maybe because we did use SMR as a tool to see if it would increase ROM directly after the treatment. But according to the theory proposed by Schleip and colleagues (2012), it would be necessary to use some kind of mobility drill directly after the SMR-session in order to obtain any increase in mobility. This is of interest during further studies that are recommended to use SMR and stretch in combination.

Another potential limitation in this study was the inability to control pressure exerted on the foam roller. A pressure carpet like the Tekscan system used by Curran et al. (2008) would have been beneficial in order to control for individual pressure variances between subjects. We did however use a numeric pain rating scale (NRS) as a tool to see if the subjects perceived pain were similar for the group. NRS is the preferred subjective pain scale for assessing pain intensity among adults (Hjermstad et al. 2011) and earlier studies have used the NRS-scale as a test to test measurement tool to control for perceived pain based on muscle soreness (MacDonald et al. 2013). You could hypothesize that the athletes' similar training schedules and training programs would put a similar strain on their bodies. This similarity should in return result in a similar muscular stiffness and fascial adhesions among the players and thereby a 27 similar pain rating within the group for each exercise as long as they put an equal pressure on the foam roller. The NRS-scale was in this case used to see if the players used an equivalent soft tissue pressure. However, the results from the NRS-scale was scattered and no conclusion could be drawn from the NRS-score. The NRS-scale is still just a subjective tool and individual variances in how the subjects perceive pain, individual soft tissue stiffness and amount of pressure put on the foam roller could explain the outcome variance. The results from the NRS showed that there was a wide variety of pain ratings (Range (0-10), m.latissimus dorsi 3-9, m.teres major/minor 2-9, thorax 0-7, m.m.rhomboideus 1-5) among the players. In order to control for any perceptual bias, only subjects who had no prior experience with SMR for the upper body were 
recruited. However, there is a possibility that the players' subjective perception of pain could have altered how they performed.

During measurements of ROM, a gravity reference inclinometer (Myrin) was used, this measurement device have been used previously and have been shown to have good intra- and interrater reliability, but only for cervical ROM (Malmström et al. 2003). There seems to be a difference in reliability depending on which joint that is being measured. Gajdosik and Bohannon (1987) discussed the fact that "reliability of measuring ROM is specific to the action measured and to regional structure and function" (Gajdosik and Bohannon, 1987). The shoulder joint is a multidirectional ball and socket joint with a large ROM-span. Even though the measurement procedure was standardized, there is a possibility that the reliability of the shoulder joint measurements are much lower compared to cervical range of motion, which in turn results in a larger measurement error. It was difficult to read the exact value from the goniometer during ROM-testing so there is a possibility that $\mathrm{a} \sim 5^{\circ}$ error margin was present during all measurements which would explain the difference in ROM between trials. Malmström et al. (2003) showed that the Myrin measurement device has got an error margin of 3.6\% to $7.6 \%$ for intratester reliability. Thus, since shoulder ROM for the different measurements varied between 29 degrees during extension up towards 189 degrees during flexion, then $3.6 \%$ to $7.6 \%$ error would equal about 1-2 degrees during extension and as much as 7-14 degrees during flexion.

Instructions to the players could have been an affecting factor, but since the participants were highly experienced players they were categorized as expert throwers and their accuracy would not have been affected by the instructions to throw as fast as possible according to earlier studies (Van den Tillaar and Ettema, 2003, 2006).

The time of 60 seconds of both SMR and DS was chosen since the aim was to see if an acute and short term session of SMR would lead to any performance enhancements. It is of interest to have a time efficient treatment that could be used shortly before game and / or during halftime. Earlier studies have used between five seconds and two minutes of SMR on each muscle without any increase in muscle activation, there has however been significant increases in ROM (Healey et al. 2014; MacDonald et al. 2013 \& Sullivan et al. 2013). An increased ROM would most likely be beneficial for handball players in order to increase throwing velocity. However, there were no significant differences in ROM among these subjects. DS still remains as the recommended and preferred specialized warm up before throwing since it does not require any equipment in comparison to SMR. This was however the first study investigating the effects of SMR on experienced athletes and further studies is needed to draw any certain conclusions regarding its 
efficiency during upper body performance on athletes

\section{Conclusion}

Based on the result of this study we conclude that an acute bout of upper body SMR does not affect ROM and does not improve throwing velocity or throwing accuracy compared to baseline or DS among elite female handball players. Neither could any correlation between ROM and throwing velocity or throwing accuracy be observed.

\section{References}

1 Awan, R., Smith, J. \& Boon, A.J (2002). Measuring Shoulder Internal Rotation Range of Motion: A Comparison of 3 Techniques. Archives of Physical Medicine and Rehabilitation 83(9): 1229-1234.

2 Barnes, M.F (1997). The Basic Science of Myofascial Release: Morphologic Change in Connective Tissue. Journal of Bodywork and Movement Therapies 1(4): 231-238.

3 Behm, D.G. \& Chaouachi, A (2011). A Review of the Acute Effects of Static and Dynamic Stretching on Performance. European Journal of Applied Physiology 111(11): 2633-2651.

4 Borsa, P.A., Laudner, K.G. \& Sauers, E.L (2008). Mobility and Stability Adaptations in the Shoulder of the Overhead Athlete: A Theoretical and Evidence-Based Perspective. Sports Medicine 38(1): 17-36.

5 Boyle, M.( 2009). Using Foam Rollers. PerformBetter. http://www.performbetter.com/webapp/wcs/stores/servlet/PBOnePieceView?storeId=10 151\& pagename=225 (Downloaded 2013-04-29).

6 Braun, S., Kokmeyer, D. \& Millett, P.J (2009). Shoulder Injuries in the Throwing Athlete. The Journal of Bone \& Joint Surgery 91: 966-978.

7 Caliskan, M. \& Akkoyunlu, Y (2011). The Evaluation of 11-15-Years-Old Footballers' Body Composition and Somatotype Features in Terms of Ball Kicking Techniques and Speed. International Journal of Sport Studies 1(4): 186-195

8 Cantu, R.I. \& Grodin, A.J. (2001). Myofascial Manipulation Theory and Clinical Application. Gaitherburg: Aspen Publishers Inc.

9 Carpenter, J.E., Blasier, R.B. \& Pellizzon, G.G (1998). The Effect of Muscular Fatigue on Shoulder Proprioception. American Journal of Sports Medicine 26: 262-265.

10 Castiglione, Angelo. (2008). Self Myofascial Release Therapy and Athletes. Australian Institute of Self Myofascial Release Therapy.

http://www.180degrees.com.au/files/self_myofascial_release_therapy_and_athletes.pdf (Downloaded 2013-04-25).

11 Cerrah, A.O., Gungor, E.O., Soylu, A.R., Ertan, H., Lees, A. \& Bayrak, C (2011). Muscular Activation Patterns During the Soccer In-Step Kick. Isokinetics and Exercise Science 19(3): 181-190.

12 Clark, M. \& Russell, A. (2009). Self-Myofascial Release Techniques. PerformBetter. http://www.performbetter.com/webapp/wcs/stores/servlet/PBOnePieceView?storeId=10 151\& catalogId=10751\&language $I d=-1 \&$ pagename=91 (Downloaded 2013-04-25). 
13 Cohen, J. (1988). Statistical Power Analysis for the Behavioural Sciences. 2nd ed. Hillsdale (NJ): Lawerence Earlbaum Associates. Curran, P.F., Fiore, R.D. \& Crisco, J.J (2008). A Comparison of the Pressure Exerted on Soft Tissue by 2 Myofascial Rollers. Journal of Sport Rehabilitation 17: 432-442.

14 Edouard, P., Degache, F., Oullion, R., Plessis, J.Y., Gleizes-Cervera, S. \& Calmels, P (2013). Shoulder Strength Imbalances as Injury Risk in Handball. International Journal of Sports Medicine 34(7): 654-660.

15 Findley, T (2009). Editorial: Fascia Research II. International Journal of Therapeutic Massage and Bodywork, 2(3): 4-9.

17 Finnoff, J.T., Newkomer, K. \& Laskowski, E.R (2002). A Valid and Reliable Method for Measuring the Kicking Accuracy of Soccer Players. Journal of Science and Medicine in Sport 5(4): 348-353.

18 Fletcher, I.M (2010). The Effect of Different Dynamic Stretch Velocities on Jump Performance. European Journal of Applied Physiology 109: 491-498.

19 Gajdosik, R.L \& Bohannon, R.W (1987). Clinical Measurement of Range of Motion Review of Goniometry Emphasizing Reliability and Validity. Physical Therapy 67(12): 1867- 1872.

20 Garcia, J.A., Sabido, R., Barbado, D. \& Moreno, F.J (2013). Analysis of the Relation Between ThrGreziosowing Speed and Throwing Accuracy in Team-Handball According to Instruction. European Journal of Sport Science 13(2): 149-154.

21 Gurinder, B (2011). Shoulder Injury in Athletes. Journal of Clinical Orthopaedics and Trauma 2(2): 85-92.

22 Grezios, A.K., Gissis, I.T.H., Sotiropoulos, A.A., Nikolaidis, D.V. \& Souglis, A.G (2006). Muscle-Contraction Properties in Overarm Throwing Movements. Journal of Strength and Conditioning Research 20(1): 117-123

23 Healey, K.C., Hatfield, D.L., Blanpied, P., Dorfman, L.R. \& Riebe, D (2014). The Effects of Myofascial Release with Foam Rolling on Performance. Journal of Strength and Conditioning Research 28(1): 61-68

24 Hjermstad, M.J., Fayers, P.M., Haugen, D.F., Caraceni, A., Hanks, G.W., Loge, J.H., Fainsinger, R., Aass, N. \& Kaasa, S (2011). Studies Comparing Numerical Rating Scales, Verbal Rating Scales, and Visual Analogue Scales for Assessment of Pain Intensity in Adults: A Systematic Literature Review. Journal of Pain and Symptom Management 41(6): 1073- 1093.

25 Klinger, W., Schleip, R., \& Zorn, A (2004). European Fascia Research Project Report. 5th World Congress Low Back and Pelvic Pain, Melbourne, Australia.

26 Kruger, K., Pilat, C., Ueckert, K., Frech, T \& Mooren, F.C (2014). Physical Performance Profile of Handball Players is Related to Playing Position and Playing Class. Journal of Strength and Conditioning Research 28(1): 117-125.

27 Leone, D.C.P.G., Pezarat, P., Valamatos, M.J., Fernandes, O., Freitas, S \& Moraes, A.C (2012). Upper Body Force Production After a Low-Volume Static and Dynamic Stretching. European Journal of Sport Science [Epub ahead of print].

28 Macdonald, G.Z., Penney, M.D.H., Mullaley, M.E., Cuconato, A.L., Drake, C.D.J., Behm, D.G. \& Button, D.C (2013). An Acute Bout of Self-Myofascial Release increases Range of Motion Without a Subsequent Decrease in Muscle Activation or Force. Journal of Strength and Conditioning Research 27(3): 812-821. 
29 MacDonald, G.Z., Button, D.C., Drinkwater, E.J. \& Behm, D.G (2014). Foam Rolling as a Recovery Tool After an Intense Bout of Physical Activity. Medicine and Science in Sports and Exercise 46(1): 131-142.

30 Malmström, E.M., Karlberg, M., Melander, A. \& Magnusson, M (2003). Zebris versus Myrin: a Comparative Study Between a Three-Dimensional Ultrasound Movement Analysis and an 31Inclinometer/Compass Method: Intradevice Reliability, Concurrent Validity, Intertester Comparison, Intratester Reliability, and Intraindividual Variability. Spine 28(21): 433-440.

31 Okamoto, T., Masuhara, M. \& Ikuta, K (2014). Acute Effects of Self-Myofascial Release Using a Foam Roller on Arterial Function. Journal of Strength and Conditioning Research 28(1): 69-73.

32 Osbahr, D.C., Cannon, D.L. \& Speer, K.P (2002). Retroversion of the Humerus in the Throwing Shoulder of College Baseball Pitchers. American Journal of Sports Medicine 30(3): 347-353.

33 Paoletti, Serge.(2006). The Fasciae Anatomy, Dysfunction \& Treatment 2ed. Seattle: Eastland Press, Inc.

34 Pieper, H.G (1998). Humeral Torsion in the Throwing Arm of Handball Players. American Journal of Sports Medicine 26(2): 247-253.

35 Póvoas, S.C., Seabra, A.F., Ascensão, A.A., Magalhães, J., Soares, J.M. \& Rebelo, A.N (2012). Physical and Physiological Demands of Elite Team Handball. Journal of Strength and Conditioning Research 26(12): 3365-3375.

36 Reagan, K.M., Meister, K., Horodyski, M.B., Werner, D.W., Carruthers, C. \& Wilk, K (2002). Humeral Retroversion and Its Relationship to Glenohumeral Rotation in the Shoulder of College Baseball Players. American Journal of Sports Medicine 30(3): 354360.

37 Ryans, I., Montgomery, A., Galway, R., Kernohan, W.G. \& McKane, R (2005). A Randomized Controlled Trial of Intra-Articular Triamcinolone and/or Physiotherapy in Shoulder Capsulitis. Rheumatology 44: 529-535.

38 Safran, M.R., Borsa, P.A., Lephart, S.M., Fu, F.M. \& Warner, J (2001). Shoulder Proprioception in Baseball Pitchers. Journal of Shoulder and Elbow Surgery 10: 438444.

39 Schleip, R., Duerselen, L., Vleeming, A., Naylor, I.L., Lehmann-Horn, F., Zorn, A., Jaeger, H. \& Klingler, W (2012). Strain Hardening of Fascia: Static Stretching of Dense Fibrous Connective Tissues can Induce a Temporary Stiffness Increase Accompanied by Enhanced Matrix Hydration. Journal of Bodywork \& Movement Therapies 16: 94-100.

40 Schleip, R (2003). Fascial Plasticity - A New Neurobiological Explanation. Journal of Bodywork and Movement Therapies 7(1): 11-19 \& 7(2): 104-116.

41 Skoufas, D., Stefanidis, P., Michailidis, C., Hatzikotoulas, K. \& Kotzamanidou, M (2003). The Effect of Handball Training with Underweighted Balls on the Throwing Velocity of Novice Handball Players. Journal of Human Movement Studies 44: 157-171.

42 Sullivan, K.M., Silvey, D.B.J., Button, B.C. \& Behm, D.G (2013). Roller-Massager Application to the Hamstrings Increases Sit-and-Reach Range of Motion Within Five to Ten Seconds Without Performance Impairments. The International Journal of Sports Physical Therapy 8(3): 228-236.

43 Threlkeld, A.J (1992). The Effects of Manual Therapy on Connective Tissue. Physical Therapy 72(12): 893-902. 
44 Torres, E.M., Kraemer, W.J., Vingren, J.L., Volek, J.S., Hatfield, D.L., Spiering, B.A., Ho, J.Y., Fragala, M.S., Thomas, G.A., Anderson, J.M.,, Häkkinen, K \& Maresh, C.M (2008). Effects of Stretching on Upper-Body Muscular Performance. Journal of Strength and Conditioning Research 22(4): 1279-1285.

45 Van Den Tillaar, R. \& Ettema, G (2003). Influence of Instruction on Velocity and Accuracy of Overarm Throwing. Perceptual and Motor Skills 96: 423-434.

46 Van den Tillar, R (2004). Effect of Different Training Programs on the Velocity of OverArm Throwing: A Brief Review. Journal of Strength and Conditioning Research 18(2): 388- 396.

47 Van den Tillaar, R. \& Ettema, G (2004). A Force-Velocity Relationship and Coordination Patterns in Over-Arm Throwing. Journal of Sports Science and Medicine 3: 211-219.

48 Van den Tillaar, R. \& Ettema, G (2007). A Three-Dimensional Analysis of Over-arm Throwing in Experienced Handball Players. Journal of Applied Biomechanics 23: 12-19.

49 Van der Wal, J (2009). The Architecture of the Connective Tissue in the Musculoskeletal System - An Often Overlooked Functional Parameter as to Proprioception in the Locomotor Apparatus. International Journal of Therapeutic Massage and Bodywork. 2(4): 1-15.

50 Vincent, W. J. \& Weir, J. P. (2012). Statistics in kinesiology, Champaign, Ill: Human Kinetics.

51 Wagner, H. \& Müller, E (2008). The Effects of Differential and Variable Training on the Quality Parameters of a Handball Throw. Sport Biomechanics 7(1): 54-71.

52 Yahia, L., Rhalmi.S., Newman, N. \& Isler, M (1992). Sensory Innervation of Human Thoracolumbar Fascia. An Immunohistochemical Study. Acta Orthopaedica Scandinavica 63(2): 195-197.

53 Zapartidis, I., Gouvali, M., Bayios, I. \& Boudolos, K (2007). Throwing Effectiveness and Rotational Strength of the Shoulder in Team Handball. Journal of Sports Medicine and Physical Fitness 47(2): 169-178.

54 Ziv, G. \& Lidor, R (2009). Physical Characteristics, Physiological Attributes, and OnCourt Performances of Handball Players: A Review. European Journal of Sport Science 9(6): 375- 386. 